Check for updates

St George's, University of London, London, UK

2 St George's University Hospitals NHS Foundation Trust, London, UK

Correspondence to: D Gill dipender.gill@nhs.net Cite this as: BMJ 2021;375:n2611 http://dx.doi.org/10.1136/bmj.n2611 Published: 28 October 2021

\title{
Safety and efficacy of antivirals against SARS-CoV-2
}

We need evidence not optimism

\author{
David B Sidebottom, 1,2 David D Smith, , 2 Dipender Gill', 2
}

In April 2021, the UK government launched an antivirals taskforce with the aim of deploying oral drugs for the prevention and treatment of covid-19 by this autumn. ${ }^{1}$ The ambitious goal raised concerns about premature adoption of new agents without sufficient evidence of their safety and efficacy. ${ }^{1}$ Nevertheless, on 20 October 2021, the UK government announced the acquisition of substantial doses of two new oral antiviral treatments for SARS-CoV-2: 480 ooo courses of molnupiravir and 250 ooo courses of PF-07321332+ritonavir. ${ }^{2}$

Molnupiravir, made by MSD (Merck), is a prodrug of ß-D-N4-hydroxycytidine, which acts as a competitive nucleoside analogue in viral RNA dependent RNA polymerase, causing multiple non-sense mutations. ${ }^{3}$ The Move-Out placebo controlled trial in over 170 sites across Latin America, Europe, and Africa evaluated the efficacy of $800 \mathrm{mg}$ molnupiravir twice daily for five days given within five days of the onset of mild or moderate covid-19 to non-hospitalised adults with at least one risk factor for severe disease..$^{-6}$ An earlier trial (Move-In) of molnupiravir in hospital inpatients was discontinued because of lack of efficacy, ${ }^{7}$ so Move-Out's inclusion criteria were modified to focus on early disease (symptom onset criterion reduced from $\leq 7$ to $\leq 5$ days) and to include only people with risk factors for severe covid-19. ${ }^{5}$

Move-Out was halted by its data monitoring committee after an interim analysis $(n=775)$ showed that molnupiravir reduced the risk of hospital admission or death from $14.1 \%$ to $7.3 \%$ (relative risk $0.55,95 \%$ confidence interval 0.36 to 0.85 , number needed to treat $=15) .{ }^{4}$ In a previous phase II trial, the same regimen of molnupiravir reduced the isolation of infectious virus on day 3 from $16.7 \%$ to $1.9 \%$ of participants $(\mathrm{P}=0.02)$, while safety events were comparable between the molnupiravir and placebo arms.

Far less data are available for Pfizer's SARS-CoV-2 protease inhibitor PF-07321332, either alone or in combination with low dose ritonavir. Ritonavir, which has an established safety profile, is commonly administered with other protease inhibitors as part of highly active antiretroviral therapy for HIV, as it inhibits hepatic metabolism of the partner drug. ${ }^{8} \mathrm{~A}$ phase I study of PF-07321332+ritonavir was completed in mid-2021 but results have not yet been published. ${ }^{9}$ Phase II and III trials are currently underway, including in high risk outpatients with symptomatic covid-19 ( $n=3000$, expected primary completion December 2021), ${ }^{10}$ low risk outpatients with symptomatic covid-19 $(\mathrm{n}=1140$, expected October 2021), ${ }^{11}$ and a trial evaluating PF-07321332+ritonavir for post-exposure prophylaxis $(n=2634$, expected December 2021). ${ }^{12}$

\section{Act on evidence, not optimism}

Limited trial data are available for both molnupiravir and PF-07321332+ritonavir. Although the recent Merck press release on molnupiravir is encouraging, ${ }^{4}$ full results from the phase III trial have not yet been made available. Questions remain about the nature of adverse events and efficacy in the context of vaccination and other treatments for covid-19. Of even greater concern, there are no publicly available safety or efficacy data for PF-07321332+ritonavir. Neither molnupiravir nor PF-07321332+ritonavir has been approved for covid-19 by any national medicines regulatory agency, although Merck has submitted an application for emergency use authorisation to the US Food and Drug Administration and has begun a rolling review with the European Medicines Agency. ${ }^{13}$

The introduction of new medicines for the prevention or treatment of covid-19 in an already strained healthcare system creates substantial complexity, especially in primary care. ${ }^{14} 15$ Ronapreve (casirivimab-imdevimab), a combination of two monoclonal antibodies for the prevention and treatment of covid-19, was introduced to UK hospitals only in September $2021^{16}$ and is still not available for outpatients. ${ }^{17}$ It remains unclear how the administration of oral antivirals for covid-19 will fit into current pathways.

In the context of persistently high morbidity and mortality attributable to covid-19, ${ }^{18}$ effective antivirals have an obvious appeal. Reduced viral load would lessen the severity and duration of disease, as well as the risk of transmitting the virus. ${ }^{19}$ However, this appeal must not cloud objective and transparent decision making in which the available evidence, resources, and clinical landscape are fully considered with decisions aimed at optimising patient care at a population level. The costs of molnupiravir and PF-07321332+ritonavir have not been publicly disclosed. Furthermore, use of antiviral monotherapies for other viruses has led to the development of resistance. ${ }^{20}$

The covid-19 antivirals taskforce was set up with the overly ambitious goal of delivering two antivirals against SARS-CoV-2 by autumn. The UK government's decision to acquire molnupiravir and PF-07321332+ritonavir in the absence of sufficient evidence of safety and efficacy raises serious concerns about further mistakes being made in an attempt to fulfil this impossible task. ${ }^{1}$

Competing interests: We have read and understood BMJ policy on declaration of interests and declare the following: DG is employed part time by Novo Nordisk and has received consultancy fees from Policy Wisdom, unrelated to the submitted work.

Provenance and peer review: Commissioned; not externally peer reviewed. 
1 Smith D, Gill D. Antivirals against SARS-CoV-2 by autumn?BM/2021;373:n1215. doi: 10.1136/bmj.n1215 pmid: 34001499

2 Department of Health and Social Care. UK government secures groundbreaking covid-19 antivirals. 2021. https://www.gov.uk/government/news/uk-government-secures-groundbreaking-covid-19antivirals

3 Painter WP, Holman W, Bush JA, etal. Human safety, tolerability, and pharmacokinetics of molnupiravir, a novel broad-spectrum oral antiviral agent with activity against SARS-CoV-2. Antimicrob Agents Chemother 2021;AAC.02428-20.pmid: 33649113

4 Merck. Merck and Ridgeback's investigational oral antiviral molnupiravir reduced the risk of hospitalization or death by approximately 50 percent compared to placebo for patients with mild or moderate covid-19 in positive interim analysis of phase 3 study. Press release, 1 Oct 2021. https://www.merck.com/news/merck-and-ridgebacks-investigational-oral-antiviral-molnupiravir-reduced-the-risk-of-hospitalization-or-death-by-approximately-50-percent-compared-toplacebo-for-patients-with-mild-or-moderat/

5 ClinicalTrials.gov. Efficacy and safety of molnupiravir (MK-4482) in non-hospitalized adult participants with covid-19 (MK-4482-002). 2020. https://clinicaltrials.gov/ct2/show/NCT04575597

6 Fischer W, Eron JJ, Holman W, etal. Molnupiravir, an oral antiviral treatment for covid-19.medRxiv 2021:2021.2006.2017.21258639.[Preprint.] doi: 10.1101/2021.06.17.21258639

7 Merck. Merck and Ridgeback Biotherapeutics provide update on progress of clinical development program for molnupiravir, an investigational oral therapeutic for the treatment of mild-to-moderate covid-19. Press release, 15 Apr 2021. https://www.merck.com/news/merck-and-ridgeback-biotherapeutics-provide-update-on-progress-of-clinical-development-program-for-molnupiravir-aninvestigational-oral-therapeutic-for-the-treatment-of-mild-to-moderate-covid-19/

8 Hull MW, Montaner JSG. Ritonavir-boosted protease inhibitors in HIV therapy. Ann Med 2011:43:375-88. doi: 10.3109/07853890.2011.572905 pmid: 21501034

9 Pfizer. Pfizer starts global phase 2/3 EPIC-PEP study of novel covid-19 oral antiviral candidate for post-exposure prophylaxis in adults. 2021. Press release, 27 Sep 2021. https://www.pfizer.com/news/press-release/press-release-detail/pfizer-starts-global-phase-23-epic-pep-studynovel-covid-19

10 ClinicalTrials.gov. A study of PF-07321332/ritonavir in nonhospitalized high risk adult participants with covid-19. 2021. https://clinicaltrials.gov/ct2/show/NCT04960202.

11 ClinicalTrials.gov. A study of PF-07321332/ritonavir in non-hospitalized low-risk adult participants with covid-19. 2021. https://clinicaltrials.gov/ct2/show/NCT05011513

12 ClinicalTrials.gov. A post-exposure prophylaxis study of PF-07321332/ritonavir in adult household contacts of an individual with symptomatic covid-19. 2021. https://www.clinicaltrials.gov/ct2/show/NCT05047601

13 Merck. Merck and Ridgeback announce initiation of a rolling review by the European Medicines Agency for molnupiravir, an investigational oral antiviral medicine, for the treatment of covid-19 in adults. Press release, 25 Oct 2021. https://www.merck.com/news/merck-and-ridgeback-announce-initiation-of-a-rolling-review-by-the-european-medicines-agency-for-molnupiravir-an-investigational-oral-antiviral-medicine-for-the-treatment-of-covid-19-in-adults/

14 Gill D, Baker EH, Hitchings AW. We need clinical guidelines fit for a pandemic. BMJ 2021;373:n1093. doi: 10.1136/bmj.n1093 pmid: 33926903

15 Park S, Elliott J, Berlin A, Hamer-Hunt J, Haines A. Strengthening the UK primary care response to covid-19. BMJ2020;370:m3691. doi: 10.1136/bmj.m3691 pmid: 32978177

16 Morgan E. "I feel special:" teen becomes one of first UK patients to receive lifesaving covid drug Ronapreve. 2021. https://www.itv.com/news/2021-09-24/teen-becomes-one-of-first-uk-patientsto-receive-lifesaving-covid-antibody-drug

17 Sidebottom DB, Gill D. Ronapreve for prophylaxis and treatment of covid-19. BM/2021;374:n2136 doi: 10.1136/bmj.n2136 pmid: 34475117

18 UK Government. Coronavirus (COVID-19) in the UK. 27 Oct 2021. https://coronavirus.data.gov.uk/

19 Burgess S, Smith D, Kenyon JC, Gill D. Lightening the viral load to lessen covid-19 severity. BMJ 2020;371:m4763. doi: 10.1136/bmj.m4763 pmid: 33303483

20 Larder BA, Darby G, Richman DD. HIV with reduced sensitivity to zidovudine (AZT) isolated during prolonged therapy. Science1989;243:1731-4. doi: 10.1126/science.2467383 pmid: 2467383

This article is made freely available for use in accordance with BMJ's website terms and conditions for the duration of the covid-19 pandemic or until otherwise determined by BMJ. You may use, download and print the article for any lawful, non-commercial purpose (including text and data mining) provided that all copyright notices and trade marks are retained. 